\title{
Physical Parameters of the IRC +10216 Circumstellar Envelope: New Constraints from Submillimeter Observations
}

\section{Citation}

Crosas, Merce, and Karl M. Menten. 1997. "Physical Parameters of the IRC +10216 Circumstellar Envelope: New Constraints from Submillimeter Observations." The Astrophysical Journal 483 (2): 913-24. https://doi.org/10.1086/304256.

\section{Permanent link}

http://nrs.harvard.edu/urn-3:HUL.InstRepos:41399806

\section{Terms of Use}

This article was downloaded from Harvard University's DASH repository, and is made available under the terms and conditions applicable to Other Posted Material, as set forth at http:// nrs.harvard.edu/urn-3:HUL.InstRepos:dash.current.terms-of-use\#LAA

\section{Share Your Story}

The Harvard community has made this article openly available. Please share how this access benefits you. Submit a story. 
THE Astrophysical Journal, 483:913-924, 1997 July 10

(C) 1997. The American Astronomical Society. All rights reserved. Printed in U.S.A.

\title{
PHYSICAL PARAMETERS OF THE IRC + 10216 CIRCUMSTELLAR ENVELOPE: NEW CONSTRAINTS FROM SUBMILLIMETER OBSERVATIONS
}

\author{
Mercè Crosas ${ }^{1}$ and Karl M. Menten ${ }^{1,2}$ \\ Received 1996 July 1; accepted 1997 February 6
}

\begin{abstract}
We have studied the temperature distribution and other physical properties of the circumstellar envelope of the prototypical high mass-loss carbon star IRC +10216 by calculating the thermal balance and the radiative transfer in the envelope self-consistently. Cooling is dominated by $\mathrm{CO}$ line emission and adiabatic expansion, and heating by dust-gas collisions throughout most of the envelope. Heating by the grain photoelectric effect is important in the outer part of the envelope. The radiative transfer is calculated by using a Monte Carlo method. The mass-loss rate, the $\mathrm{CO}$ abundance, the dust-gas momentum transfer efficiency, and the distance to the source are free parameters in our model. These physical parameters are constrained by the comparison of our model results with the observations of various ${ }^{12} \mathrm{CO}$ and ${ }^{13} \mathrm{CO}$ lines. In particular, recent submillimeter-wavelength observations of moderately high excitation transitions, such as the $J=6 \rightarrow 5$ line, put very important constraints on the temperature distribution in the inner part of the envelope, and they do not support the presence of very high temperatures $(T \sim 500-1000 \mathrm{~K})$ in the inner part of the envelope (at about $5 \times 10^{15} \mathrm{~cm}$ from the central star) suggested by a previous study. We also find that a mass-loss rate of $3.25 \times 10^{-5} M_{\odot} \mathrm{yr}^{-1}$ and a distance of $150 \mathrm{pc}$ provide the best agreement between our model results and observations.

Subject headings: circumstellar matter — radio lines: stars - stars: AGB and post-AGB stars: individual (IRC +10216) - stars: mass loss
\end{abstract}

\section{INTRODUCTION}

Late-type stars lose significant amounts of their mass once they have left the main sequence. Studying this socalled asymptotic giant branch (AGB) phase of their evolution provides important information on the transition from red giant stars to planetary nebula, and on the amount and chemical composition of the material returned to the interstellar medium (ISM) (see, e.g., Zuckerman 1980 and Jura \& Kleinmann 1989). Given the high density of the circumstellar material and the low temperatures of the stellar photosphere, the material ejected by cool giant stars is mainly in molecular form. Once the gas reaches a certain distance from the star, the dust condenses, and the radiation pressure starts accelerating the dust that transfers momentum to the gas, thus forming an expanding envelope around the star.

The prototypical carbon-rich mass-losing giant star is IRC +10216 (CW Leo). Given its small distance, $d$, of less than 200 pc (Kwan \& Hill 1977) and its prodigious massloss rate, estimated in previous studies to be between 2 and $4 \times 10^{-5} M_{\odot} \mathrm{yr}^{-1}$ (Kwan \& Linke 1982; Truong-Bach, Morris, \& Nguyen-Q-Rieu 1991), many phenomena can be studied toward IRC + 10216 in great detail that are difficult or impossible to observe toward other carbon stars. In particular, more than 50 molecular species have been detected in its envelope at radio and infrared wavelengths (e.g., Johansson et al. 1984; Avery et al. 1992; Groesbeck, Phillips, \& Blake 1994; Kawaguchi et al. 1995). After $\mathrm{H}_{2}$, which is difficult to observe, $\mathrm{CO}$ is the most abundant molecule. Its abundance remains constant throughout most of the envelope (Huggins \& Glassgold 1982). Thus, CO is formed

\footnotetext{
${ }^{1}$ Harvard-Smithsonian Center for Astrophysics, 60 Garden Street, Cambridge, MA 02138

${ }^{2}$ Present address: Max-Planck-Institut für Radioastronomie, Auf dem Hügel 69, D-53121, Bonn, Germany.
}

in the photosphere of the star by LTE gas-phase reactions, and it is not destroyed until photodissociation by external UV radiation takes place in the outer layers of the envelope (Mamon, Glassgold, \& Huggins 1988).

Observations of $\mathrm{CO}$ rotational emission at millimeter and submillimeter wavelengths can be used to determine the kinetic temperature distribution in the circumstellar envelope and to derive the mass-loss rate of the star. Knowledge of the temperature distribution is necessary to interpret the observed emission of other molecular species, and knowledge of the mass-loss rate is essential for understanding the AGB transitional phase. The temperature distribution and the mass-loss rate can be obtained only by solving the radiative transfer problem self-consistently with the thermal balance. In these calculations, the mass-loss rate, the $\mathrm{CO}$ abundance, the distance to the source, and the dust properties are adjustable parameters.

Kwan \& Linke (1982) used this procedure, and their model suggested a mass-loss rate, $\dot{M}$, of $4 \times 10^{-5} M_{\odot} \mathrm{yr}^{-1}$ and a dust-to-gas momentum transfer efficiency, $Q$, of $2.35 \times 10^{-2}$, when it was compared with observations of the CO $J=1 \rightarrow 0$ and $J=2 \rightarrow 1$ emission lines. These results were found for a distance $d=200 \mathrm{pc}$ and a CO abundance with respect to molecular hydrogen, $\left[\mathrm{CO} / \mathrm{H}_{2}\right]$, of $6 \times 10^{-4}$. However, their model did not reproduce the shape of the CO $J=1 \rightarrow 0$ line profile; it predicted a double-peaked line contrary to the observed flat spectrum. Furthermore, their model predicted a radial distribution of the main-beam brightness temperature at $\mathrm{CO} J=1 \rightarrow 0$ line center that failed to reproduce the observed radial distribution in the inner and outer parts of the envelope: the line-center temperature from their model was higher than that observed in the inner part and lower in the outer part. Huggins, Olofsson, \& Johansson (1988) then included grain photoelectric heating caused by the external ultraviolet field in order to increase the temperature in the outer part of the 
envelope. They also discussed the photodissociation of $\mathrm{CO}$ in the outer envelope. This, together with lower values for the hydrogen mass-loss rate $\left(\dot{M}=2.5 \times 10^{-5} M_{\odot} \mathrm{yr}^{-1}\right)$ and for the dust momentum transfer parameter $\left(Q=1.5 \times 10^{-2}\right)$, improved the agreement between model and observations. On the other hand, Sahai (1987) suggested a two-shell model to account for the differences between the inner and the outer envelope. Truong-Bach et al. (1991) used a two-shell model, as proposed by Sahai (1987), in which the mass-loss rate and the momentum transfer parameter changed at a radius of about $6 \times 10^{16}$ $\mathrm{cm}$ (for $d=200 \mathrm{pc}$ ). To constrain their model calculations, they used a CO $J=6 \rightarrow 5$ observation published by Koepf et al. (1982), which was taken at the Infrared Telescope Facility with a $35^{\prime \prime}$ beam, and the data for the CO $J=3 \rightarrow 2, \quad J=2 \rightarrow 1$, and $J=1 \rightarrow 0$ transitions were obtained with various telescopes. The resulting model of the temperature distribution predicts very high temperatures in the inner envelope region, about $1000 \mathrm{~K}$ at $2^{\prime \prime}$ radius, which is about an order of magnitude higher than the temperature predicted by the Kwan \& Linke (1982) model at that radius. It is clear that the interpretation of observations of molecular lines originating from the inner part of the envelope, e.g., column density estimates, will be dramatically different depending on whether the kinetic temperature distributions predicted by Truong-Bach et al. or Kwan \& Linke are used.

In this paper, we present new model calculations that are aimed at fitting published $\mathrm{CO}$ line profiles and maps, as well as new high spatial resolution submillimeter $\mathrm{CO}$ observations of the $J=6 \rightarrow 5$ transition. We reexamine the possible values for the mass-loss rate, the $\mathrm{CO}$ abundance, the dust-to-gas momentum transfer efficiency, and the distance of IRC + 10216 and obtain a temperature profile that best fits the observations. Our new $\mathrm{CO} J=6 \rightarrow 5$ data have $4-5$ times higher spatial resolutions than previously published data for this line, and thus they provide stringent new information on the temperature distribution within the inner $10^{16} \mathrm{~cm}$ of the envelope, a region poorly constrained by all previous $\mathrm{CO}$ studies. We use a more complete and updated model for the $\mathrm{CO}$ emission than previous efforts. Among other things, (i) we consider a much larger number of rotational levels $\left(J_{\max }=30\right)$ for $\mathrm{CO}$, which is necessary to determine realistically the radiative cooling in the inner region, (ii) we include cooling by ${ }^{13} \mathrm{CO}$ transitions, and (iii) we use recently published revised $\mathrm{CO}$ collisional coefficients. Instead of employing the more commonly used large velocity gradient (LVG) approximation, we treat the radiative transfer in the envelope with a Monte Carlo method. This allows consideration of a finite turbulent velocity field in the radiative transfer calculations, which is impossible in LVG models. Thus, it can be used to verify whether or not the LVG approximation holds for circumstellar envelopes. In addition, contrary to LVG models, with a Monte Carlo model it is straightforward to consider radial variations of physical parameters, such as the gas velocity, and it is possible to perform the calculations for arbitrary envelope geometries.

Our radiative transfer model is described in $\S 2$. In $\S 3$, we define the contributions to the heating and cooling in the circumstellar envelope used in our thermal balance model. The observations used to constrain our physical parameters are described in $\S 4$. In $\S \S 5$ and 6, we present and discuss the model results, and compare them with the observations and previous modeling efforts. A short summary is given in $\S 7$.

\section{RADIATIVE TRANSFER}

\subsection{Description of the Model}

We solved the radiative transfer for ${ }^{12} \mathrm{CO}$ and ${ }^{13} \mathrm{CO}$ in the expanding envelope by using a Monte Carlo code published by Bernes $(1978,1979)$, which we modified and updated as described below. In the Monte Carlo simulation, model photons $\left(10^{3}-10^{4}\right)$, which represent a large number of real photons, are followed throughout the cloud until they escape. The absorption and the stimulated emission terms for the statistical balance equation are computed as the number of photons decreases because of absorptions by the molecules they encounter along their path. A pseudorandom number generator is used to assign an initial position, a direction, and a frequency (within the corresponding passband of the line) to each model photon. These model photons are mainly photons emitted inside the envelope, but we also consider photons from the cosmic microwave background, which has a temperature, $T_{\mathrm{CMB}}$, of $2.73 \mathrm{~K}$, and millimeter-wavelength photons from the central star, for which we assume a temperature, $T_{*}$, of $2000 \mathrm{~K}$.

The region is divided into spherical shells, with a minimum radius of $r_{\min }=5 \times 10^{14} \mathrm{~cm}\left(0^{\prime \prime} .22\right.$ if $\left.d=150 \mathrm{pc}\right)$ and a maximum radius of $r_{\max }=3.75 \times 10^{17} \mathrm{~cm}\left(170^{\prime \prime}\right.$ if $d=150 \mathrm{pc}$ ), in such a way that the temperature, the density, and the molecular abundance can be considered constant within the shell and statistical balance applies in each shell. However, these physical conditions may vary from one shell to another. The CO abundance is assumed to be constant throughout the envelope and then decreases at the outer radius because of photodissociation. The density varies as $\left[r^{2} v(r)\right]^{-1}$. The expansion velocity of the gas, $v(r)$, increases in the inner region of the envelope, where the dust is still condensing, monotonically with radius $r{ }^{3}$ As the dust forms, stellar radiation provides the pressure necessary to accelerate the dust. The gas is accelerated by gaining momentum from collisions with the dust grains. This acceleration has been commonly assumed to be (e.g, TruongBach et al. 1991)

$$
v(r)=v_{\infty}\left(1-\frac{\theta_{0}}{\theta}\right)^{1 / 2},
$$

where $\theta_{0}$ is the characteristic radius for dust formation and has a value of $00^{\prime \prime} 133$ (Kwan \& Linke 1982). The radial dependence of $v$ given in equation (1) reproduces satisfactorily the velocity increase in the region where the dust forms. The equation also gives the correct asymptotic behavior in the outer region of the envelope, with the velocity approaching the terminal velocity $v_{\infty}$. With knowledge of the dust opacity and using the equation of motion of the ejected gas, in principle one can calculate the velocity acceleration (see Goldreich \& Scoville 1976). However, in the region where the dust forms, the opacity changes with radius in a complicated way that is little understood at present. The acceleration law described by equation (1) certainly represents a crude approximation. We adopt it for the sake of simplicity and justify its use by noting that, because of beam dilution, the $\mathrm{CO}$ emission emerging from regions where $v<v_{\infty}$ will contribute only a very small

\footnotetext{
${ }^{3}$ We will use $r$ to denote the linear radius from the center of the star in centimeters and $\theta$ to denote the corresponding angular radius in arcseconds, assuming a distance to IRC +10216 of $150 \mathrm{pc}$.
} 
portion of the emission observed in any of the lines discussed in this paper.

The kinetic temperature is obtained as a function of radius by solving the thermal balance problem as discussed in $\S 3$. The radiative transfer and the thermal balance are calculated in an iterative fashion until convergence in the temperature profile is achieved (see $\S 3.4$ ).

\subsection{Infrared Pumping, Collisions, and Radiative Transitions}

We consider infrared pumping due to emission from the dust heated by stellar radiation. Following the example of Toombs et al. (1972), previous studies of CO infrared excitation in the IRC +10216 circumstellar envelope have described the infrared emission at $4.6 \mu \mathrm{m}$ (the wavelength corresponding to the first vibrationally excited state of $\mathrm{CO}$ ) by a dust distribution of radius 0.2 and a blackbody temperature of $T_{d} \approx 600 \mathrm{~K}$ (e.g., Kwan \& Linke 1982; TruongBach et al. 1991). Recently, it has been shown that the infrared data is better modeled by using a continuous distribution for the dust temperature (Ivezić \& Elitzur 1996) rather than by using discrete dust shells, as proposed first by Toombs et al. (1972) and later by Sloan \& Egan (1995). However, the emission from this continuous distribution is emerging from the inner 2" region only. For the purpose of our work, the details of the infrared field in this inner region are irrelevant, and the above approximation $\left(T_{d} \approx 600 \mathrm{~K}\right.$ blackbody of radius 0.2 ), which produces practically the same flux at $4.6 \mu \mathrm{m}$ in the region of interest as that given by Ivezić \& Elitzur (1996), is adequate. In $\S 6.4$, we shall discuss the validity of our approximation in more detail.

To include infrared pumping, it is assumed that absorptions resulting in excitations to the first vibrational state decay immediately back to the ground state, so that the population in this excited state is negligible. This assumption is valid because, under the physical conditions within the envelope, the $v=1$ state has no time to distribute its population among its rotational levels before a spontaneous decay to $v=0$ (with Einstein $A$ values between 20 and $30 \mathrm{~s}^{-1}$ ) takes place. Thus, a vibrational excitation may effectively produce a change of the rotational state in the ground level and therefore can be treated effectively as a collisional excitation in the statistical balance equations (Deguchi \& Uyemura 1984). The terms representing net excitations $\left(R_{J-1, J+1}\right)$ or de-excitations $\left(R_{J+1, J-1}\right)$ can be expressed in the following way:

$$
\begin{aligned}
& R_{J-1, J+1}=\frac{2 J+1}{2 J-1} A_{R} \frac{A_{P}}{A_{P}+A_{R}} \rho_{R}, \\
& R_{J+1, J-1}=\frac{2 J+1}{2 J+3} A_{P} \frac{A_{R}}{A_{P}+A_{R}} \rho_{P},
\end{aligned}
$$

where $A_{R}$ and $A_{P}$ are the spontaneous decay probabilities for the $R$-branch (from $v=1$ with $J$ to $v=0$ with $J-1$ ) and the $P$-branch (transitions from $v=1$ with $J$ to $v=0$ with $J+1)$, respectively. The values are taken from Goorvitch \& Chackerian (1994). The $\rho_{R}$ and $\rho_{P}$ are defined as

$$
\rho_{i}=\frac{W \exp \left(-\tau_{i}\right)}{1-\exp \left(h v_{i} / k T_{d}\right)},
$$

where $i=R$ or $P$, and the optical depth is approximated as $\tau=(h v / 4 \pi \Delta v) n_{l} B_{l u} \Delta s$. In the above equations, $T_{d}$ is the dust temperature, $h$ is the Planck constant, $v$ is the photon frequency, $k$ is the Boltzmann constant, $n_{l}$ is the population of the lower level, $B_{l u}$ is the Einstein $B$-coefficient, $\Delta v$ is the Doppler width, and $\Delta s$ is the distance traveled in the envelope along the line of sight. Stimulated emissions from the vibrationally excited state to the ground state are ignored because the population in the $v=1$ state is very small. In summary, the infrared pumping terms in equations (2) and (3) consider absorptions to the $v=1$ state due to the infrared field, including the geometrical dilution and attenuation of the field, and spontaneous decays to the $v=0$ ground state.

Infrared pumping can be very important in cases where the molecular abundance is low (for abundances of less than $10^{-6}$; see, for example, the case of $\mathrm{HC}_{5} \mathrm{~N}$ described by Deguchi \& Uyemura 1984) or where the density is low (for mass-loss rates of less than $10^{-7} M_{\odot} \mathrm{yr}^{-1}$, as shown in Morris 1980). In our case, however, infrared pumping is not a dominant term in the statistical balance of $\mathrm{CO}$ because most of the infrared radiation is absorbed by this abundant molecule very close to the inner surface of the envelope, within the first subshell that has a radius of $5.5 \times 10^{14} \mathrm{~cm}$. We find that even ${ }^{13} \mathrm{CO}$, which is less abundant than ${ }^{12} \mathrm{CO}$ by a factor of about 50 (Kahane et al. 1992), is optically thick to the infrared radiation within $r \approx 5 \times 10^{15} \mathrm{~cm}$. Thus, the important terms in the radiative transfer calculations are collisional excitations and de-excitations, spontaneous decays, and absorptions and stimulated emissions due to millimeter-wavelength photons. To interpolate the collisional rate coefficients over the range of temperatures considered, we fitted an empirical function of the form given by de Jong, Chu, \& Dalgarno (1975) to the rates calculated by Flower \& Launay (1985). We use 30 rotational levels for the radiative transfer of ${ }^{12} \mathrm{CO}$ and ${ }^{13} \mathrm{CO}$ each.

\subsection{Details of the Monte Carlo Method}

As mentioned above, the terms representing the absorptions and stimulated emissions in the statistical balance equation are calculated by using a Monte Carlo simulation in the following way. A large number of real photons is represented by a model photon with an initial weight of one. There are three different sources of model photons: those emitted by the $\mathrm{CO}$ molecules in the envelope, those emitted by the central star, and those coming from the background. The model photons emitted by $\mathrm{CO}$ are assigned at random a starting position inside the envelope, a direction, and a frequency within the line profile, which is assumed to be a Gaussian. The stellar and the background model photons start their trajectory from the inner and the outermost envelope boundary, respectively, and are assigned a random direction. Their number and frequency are given by the blackbody curve corresponding to the stellar and the background temperature, respectively. The photons travel from their initial position to the closest shell boundary along the assigned direction. However, if the corresponding distance is larger than a maximum step size, then the photons are forced to stop after this maximum step. In the original Bernes (1978) Monte Carlo code, the value for the maximum step is constant and is given as an initial parameter. Here we define this value as a function of position; it must be satisfied at any given radius that the change in $v(r)$ $\cos \mu$ cannot be larger than about $10 \%$ of the Doppler velocity width, where $\mu$ is the angle between the direction of the photon and the outward radial direction. If a photon traveled a larger distance than our maximum step, then, in the new position, the molecules would have a very different 
velocity than the molecules in the initial position, so the photon would not be absorbed in the new position. However, if the density is high around the emitting position, the photon should have been absorbed along its path. Therefore, if we allowed the photons to travel larger distances than our maximum step, the absorption term, which is computed at the new position, could be underestimated. After each step, the model photons reduce their initial weight because of the attenuation by $\mathrm{CO}$ absorptions. The model photons are traced until they escape from the envelope. By following this process for all model photons, we obtain a value for the absorption term, and consequently the stimulated emission term, for each shell and each CO rotational transition.

In the first iteration in which this procedure is applied, the level populations are those given by local thermodynamic equilibrium. Once we obtain the absorption and the stimulated emission terms, after we have followed all of the model photons, we can solve the statistical balance equations for each shell. Then we use the new level populations to repeat the simulation in order to obtain more realistic values for the absorption and emission terms. This process is applied as many times as necessary to reach convergence, which is typically achieved after 15 iterations. The final result for the absorption and the emission terms of the statistical balance equation are actually the average values over the last few iterations. This is practically equivalent to using the mean value of different Monte Carlo runs, because every time the above procedure is applied, we use a different random number generator result to assign the position, direction, and frequency of the model photons.

In the Bernes code, the background, stellar, and envelope photons are redefined relative to a uniform reference field for each transition. This helps to reduce the numerical noise inherent to a Monte Carlo method. However, this reference field needs to be chosen so that its temperature is similar to the excitation temperature of the transition. While this can be implemented easily if the whole region of interest can be described approximately with one excitation temperature (as is the case for the isothermal interstellar cloud considered by Bernes 1979), the usage of a reference field is not straightforward in our case of an envelope in which the excitation conditions change substantially with radius. Consequently, we do not use a reference field in our calculations and simply use a greater number of model photons in the simulation in order to reduce the Monte Carlo noise. Even if this increases the running time, a typical Monte Carlo run using a Sun SPARC 1000 takes only about 5 minutes total time. We choose the number of model photons depending on the number of shells in which the region is divided; the number of photons per shell is usually between 50 and 100, and the number of shells is between 30 and 40 for our model circumstellar envelope.

One of the advantages of a Monte Carlo simulation is that any turbulent velocity in the region of interest can be easily considered in the model. This is not the case for calculations using the LVG approximation, where the turbulent velocity is assumed to be negligible in the statistical balance calculations. We consider the effect of a turbulent velocity of $1 \mathrm{~km} \mathrm{~s}^{-1}$. To check our code, we compared the results of our calculations with the results produced with the LVG code used by Bieging \& Tafalla (1993). By using the same input parameters, we found that both models produced remarkably similar results for the excitation temperature and the CO line profiles. This allowed us to confirm the validity of our radiative transfer model and, at the same time, to show that there is no difference between the LVG approximation and the Monte Carlo simulation in the case of IRC +10216 . In the future, we intend to compare our code with the LVG code for other cases in which the large velocity gradient approximation is questionable.

In addition, we compared the results of our calculations with those of Morris (1980) in order to check our treatment of the infrared pumping in the radiative transfer calculations. We ran four cases with the different mass-loss rates discussed in Morris' paper. In these runs, instead of using the Flower \& Launay (1985) collisional rate coefficients, we used the "older" values calculated by Green $\&$ Thaddeus (1976), which were used by Morris. We find perfect agreement between our results and Morris's results for all four cases considered: the excitation temperatures of $\mathrm{CO}$ $J=1 \rightarrow 0$ and CO $J=2 \rightarrow 1$ show the same behavior in both models, with differences of less than $5 \%$.

\section{THERMAL BALANCE}

In carbon-rich circumstellar envelopes, the thermal balance is controlled mainly by gas-dust collisional heating, adiabatic cooling, and ${ }^{12} \mathrm{CO}$ radiative cooling in most parts of the envelope (e.g., Kwan \& Linke 1982). In the outer region, dust photoelectric heating can be important (Huggins et al. 1988), and, as discussed in $\S 5.1,{ }^{13} \mathrm{CO}$ can be a significant coolant in some parts of the envelope. Recent observations with the Infrared Space Observatory (ISO) (Cernicharo et al. 1996) suggest that $\mathrm{HCN}$ is also a very important coolant in the inner part of the IRC +10216 envelope and, in fact, might be the dominant coolant at radii $\leq 10^{15} \mathrm{~cm}$, due to the large amount of far-infrared lines arising from different vibrational levels of $\mathrm{HCN}$. Exploration of the temperature distribution within this innermost region is outside the scope of the present study, which concerns itself with the parts of the envelope that are at radii larger than a few times $10^{15} \mathrm{~cm}$. The angular size of the inner region is much smaller than that of the region giving rise to the bulk of the low- $J$ CO lines and much smaller than the telescope beamwidths involved in the observations modeled by us. We estimate that uncertainties in the temperature distribution within a radius of $10^{15} \mathrm{~cm}$ will introduce uncertainties in our model line profiles that, at most, are of order a few percent of the total emission in the $J \leq 6$ CO lines.

\subsection{Dust-Gas Collisional Heating}

The heating due to dust-gas collisions, $H_{\mathrm{d}-\mathrm{g}}$, can be expressed as

$$
\begin{aligned}
H_{\mathrm{d}-\mathrm{g}} & =\frac{1}{2} \rho_{\mathrm{H}_{2}} v_{d}^{3} n_{d} \pi\left\langle a_{d}\right\rangle^{2}, \\
& =\frac{3}{8} m_{\mathrm{H}_{2}}^{2} n_{\mathrm{H}_{2}}^{2} \frac{\delta}{\rho_{d}\left\langle a_{d}\right\rangle} v_{d}^{3} \operatorname{ergs~cm}^{-3} \mathrm{~s}^{-1},
\end{aligned}
$$

where $\rho_{\mathrm{H}_{2}}, m_{\mathrm{H}_{2}}$, and $n_{\mathrm{H}_{2}}$ are the molecular hydrogen mass, number density, and mass density, respectively, $n_{d}$ is the dust number density, $\delta$ is the dust-to-gas mass ratio, $\rho_{d}$ is the density of a dust grain, $\left\langle a_{d}\right\rangle$ is the average size of the dust grain, and $v_{d}$ is the drift velocity of the dust with respect to the gas. To obtain the drift velocity, we consider the contribution resulting from radiation pressure, $v_{\text {rad }}$, and then correct for the thermal velocity, $v_{\text {therm }}$, which can be 
relevant in the hotter, inner region. We use the formula proposed by Kwok (1975),

$$
v_{d}=v_{\mathrm{rad}}\left[\left(1+x^{2}\right)^{1 / 2}-x\right]^{1 / 2},
$$

where $x=0.5\left(v_{\text {therm }} / v_{\text {rad }}\right)^{2}$, and

$$
\begin{aligned}
v_{\mathrm{rad}} & =\left(\frac{L v Q}{\dot{M} c}\right)^{1 / 2}, \\
v_{\text {therm }} & =\frac{3}{4}\left(\frac{3 k T_{K}}{m_{\mathrm{H}_{2}}}\right)^{1 / 2} .
\end{aligned}
$$

Here $L$ is the stellar luminosity, $v$ is the gas velocity, which changes with radius in the dust condensation region (see $\S 2.1$ ), and $T_{K}$ is the kinetic temperature. The stellar luminosity has a value of $2.1 \times 10^{4} L_{\odot}\left(d / d_{0}\right)^{2}$ (Becklin et al. 1969), where $d_{0}=200$ pc. $Q$ is the dust momentum transfer parameter.

It has been suggested recently that dust grains in the IRC +10216 circumstellar envelope have a distribution of sizes (Ivezić \& Elitzur 1996; Jura 1994). For example, Jura (1994) proposes a distribution that can account for the ultraviolet opacity derived from observations, which requires small grains $(\sim 0.015 \mu \mathrm{m})$, and for the near-infrared polarization, which requires large grains $(\geq 0.1 \mu \mathrm{m})$. The mean value of this distribution is $\sim 0.05 \mu \mathrm{m}$. This value has been commonly used as the typical value for the size of amorphous carbon grains, which dominate in the IRC +10216 envelope (Lorenz-Martins \& Lefévre 1993; Habing, Tignon, $\&$ Tielens 1994). We used this mean value in equation (5), instead of considering a size distribution that would require integration of the equation over the grain size. To do this integration correctly, we would need to include the dependence of $Q$ on $a_{d}$. For the sake of simplicity, we "absorb" the effect of using an average grain size as opposed to a grain size distribution in the value of the parameter $Q$, so that equation (5) does not need to be modified.

The density of a dust grain is taken to be $\rho_{d}=1.85 \mathrm{~g}$ $\mathrm{cm}^{-3}$ (Rouleau \& Martin 1991), and the value for the dustto-gas mass ratio is chosen to be the same as the typical value in the ISM, i.e., $\delta=0.01$.

\subsection{Radiative Cooling}

Calculating the radiative cooling due to $\mathrm{CO}$ rotational lines is straightforward once the level populations are known. It can be expressed in terms of the spontaneous decays, the stimulated emissions, the absorptions, and the infrared pumping rates. However, by using the statistical balance equations, it also can be expressed in terms of the collisional excitations $\left(C_{i j}\right)$ and de-excitations $\left(C_{j i}\right)$ only, in a more general and convenient way:

$$
C_{\mathrm{CO}}=\sum_{k=1}^{n}\left(\sum_{i<k, j \geq k} n_{i} C_{i j}-n_{j} C_{j i}\right) h v_{k k-1} \operatorname{ergs~cm}^{-3} \mathrm{~s}^{-1} .
$$

In this equation, $n_{i}$ and $n_{j}$ are the level population of a lower and an upper level, respectively, and $v_{k k-1}$ is the frequency of a transition from a rotational level $k$ to $k-1$. A total of $n=29$ transitions are considered. The same formula applies to ${ }^{13} \mathrm{CO}$. In the inner region, where $r$ is a few times $10^{15} \mathrm{~cm}$, emission from rotational levels as high as $J \geq 25$ dominates the cooling; they therefore must be considered to calculate the radiative cooling correctly in this hot, dense part of the envelope. At larger radii, $r \geq 10^{16} \mathrm{~cm}$, emission from transitions between levels with $J<10$ dominates the cooling.

\subsection{Photoelectric Heating}

We also include the heating caused by electrons ejected from the dust grains by external UV photons. We use the formula given by Draine (1978), which can be reduced to a constant multiplied by the gas number density, i.e.,

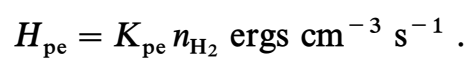

There is some degree of uncertainty in the value of the constant $K_{\mathrm{pe}}$ that depends on the dust properties. For standard grains, its value in the outer part of the envelope is approximately $K_{\mathrm{pe}} \approx 1 \times 10^{26} \mathrm{ergs}^{-1}$ (Huggins et al. 1988). But it can be a factor of 2 or 3 larger for amorphous carbon, and even an order of magnitude larger for very small grains (Jura 1976). We choose a value of $K_{\mathrm{pe}}=$ $2 \times 10^{26}$ ergs s$^{-1}$, which is consistent with the mean grain size in the envelope.

\subsection{Thermal Equilibrium Equation}

With the heating and cooling terms determined, we can calculate the gas temperature by solving the differential equation following from the first law of thermodynamics for an expanding gas (e.g., Goldreich \& Scoville 1976),

$$
\frac{d T}{d r}=(2-2 \gamma)\left(1+0.5 \frac{d \ln v}{d \ln r}\right) \frac{T}{r}+\frac{\gamma-1}{n_{\mathrm{H}_{2}} k v}(H-C) .
$$

Here $\gamma$ is the adiabatic index, $n_{\mathrm{H}_{2}}$ is the $\mathrm{H}_{2}$ number density, and $H$ and $C$ are the total heating and cooling rates in units of ergs $\mathrm{s}^{-1} \mathrm{~cm}^{-3}$. The first term on the right-hand side of equation (11) corresponds to the adiabatic cooling. We use a value of $\gamma=5 / 3$, which is correct for molecular hydrogen at temperatures lower than $300 \mathrm{~K}$, for which its rotational excitation is negligible. This is true in most parts of the envelope, for $r \geq 2 \times 10^{15} \mathrm{~cm}$, where the comparison of our model with observations is relevant. In the innermost region, the variation from $\gamma=5 / 3$ to $\gamma=7 / 2$ affects the results insignificantly $(\leq 3 \%)$ (Groenewegen 1994$)$.

In equation (11), the molecular radiative cooling, $C$, depends implicitly on the temperature because of radiative transfer effects. Therefore, we need to choose an initial temperature profile to solve the radiative transfer problem, and then use the level populations given by this initial temperature to calculate $C_{12 \mathrm{CO}}$ and $C_{13 \mathrm{CO}}$, the cooling contributions of ${ }^{12} \mathrm{CO}$ and ${ }^{13} \mathrm{CO}$. Instead of guessing an initial temperature profile, in some cases we first solve the thermal balance without including the radiative cooling in order to obtain an approximate temperature to use in the first iteration. The temperature at the first point of our calculations, $\theta_{\min }=0 \prime 22$, is taken to be $1200 \mathrm{~K}$. Substituting the heating and cooling terms in equation (11), we then obtain numerically a new temperature as a function of radius. We calculate as many iterations between the radiative transfer and the thermal balance as needed to achieve convergence in the temperature profile. Usually, as many as 70 iterations are required to find a temperature profile that does not vary significantly $(\sim 0.5 \%)$ from one iteration to the next within the Monte Carlo noise. Since the convergence is so slow, we need to verify that we in fact have found a unique solution for the temperature distribution. This was done by varying the initial temperature profile significantly. In all cases, our calculations produced the same final temperature profile regardless of the initial distribution, except near $\theta_{\min }=$ 0 ".22, where the results depend on the arbitrarily given temperature at this point. 


\section{OBSERVATIONS}

We use observations of the ${ }^{12} \mathrm{CO} J=1 \rightarrow 0, J=2 \rightarrow 1$, $J=3 \rightarrow 2, J=4 \rightarrow 3$, and $J=6 \rightarrow 5$ transitions and the ${ }^{13} \mathrm{CO} J=1 \rightarrow 0$ and $J=6 \rightarrow 5$ transitions to compare with our theoretical results and thus constrain the model parameters.

New observations of ${ }^{12} \mathrm{CO} \quad J=6 \rightarrow 5$ and ${ }^{13} \mathrm{CO}$ $J=6 \rightarrow 5$ obtained with the James Clerk Maxwell Telescope (JCMT) and the Caltech Submillimeter Observatory (CSO) were kindly provided to us by A. Harris (JCMT data) and $\mathrm{K}$. Young (CSO data). Data acquisition and calibration procedures at the JCMT and CSO were similar to those described by Harris et al. (1995) and Menten \& Young (1995), respectively, and the receivers used are described by Harris et al. (1994) and Kooi et al. (1994).

The JCMT data set consisted of a spectrum taken toward the position of IRC +10216 , as well as of spectra taken at 8 " offset from the stellar position along the cardinal directions. In addition, one spectrum was taken toward a position $16^{\prime \prime}$ north of the star. We averaged the four spectra taken at $8^{\prime \prime}$ offsets to increase the signal-to-noise ratio and compare our model result with this average spectrum. According to Harris et al. (1995), the JCMT at the frequency of the CO $J=6 \rightarrow 5$ transition $(691.473 \mathrm{GHz})$ has $40 \%$ of the power in a compact beam of $7 "$ FWHM and $60 \%$ of the power in a component with $20^{\prime \prime}$ FWHM. The CSO $J=6 \rightarrow 5$ spectrum is taken toward the stellar position with a beam size of about $10^{\prime \prime}$ FWHM.

For the other lines, we use a number of published observations taken with different beam sizes. For the $\mathrm{CO}$ $J=1 \rightarrow 0$ line, we use observations of the on-source line profile obtained with the IRAM $30 \mathrm{~m}$ telescope and maps obtained with the IRAM, NRAO $12 \mathrm{~m}$, and Bell Telephone Laboratories (BTL) $7 \mathrm{~m}$ telescopes. We also use a CO $J=2 \rightarrow 1$ line profile and radial distribution in that line measured with the IRAM telescope as well as the radial distribution observed with the NRAO telescope. For the CO $J=3 \rightarrow 2$ and the CO $J=4 \rightarrow 3$ transitions, we use observations obtained with the JCMT. For the CO $J=3 \rightarrow 2$ line, the radial emission distribution was published, while for the CO $J=4 \rightarrow 3$ line, only the onsource line profile was available. We also use observations of the ${ }^{13} \mathrm{CO} J=1 \rightarrow 0$ line obtained with the IRAM telescope. In this case, we compare our model results with published spectra taken at offsets of $0^{\prime \prime}, 10^{\prime \prime}$, and $20^{\prime \prime}$ from the star.
A summary of the observations described above is given in Table 1, together with references. We note that uncertainties in the absolute calibration of millimeter-wavelength and, in particular, submillimeter-wavelength observations are typically of order $20 \%$ or more.

\section{MODEL RESULTS}

The objective is to explore the effect of variations in the physical parameters on the results of the radiative transfer and the thermal balance model, and to find a set of parameter values that best reproduces the observations. In our calculations, the mass-loss rate, the $\mathrm{CO}$ abundance, the distance to the source, and the dust properties are varied.

\subsection{Radiative Cooling Results}

The radiative cooling depends on the $\mathrm{CO}$ level populations and on the gas (molecular hydrogen) density. This means that changes in the mass-loss rate, the $\mathrm{CO}$ abundance, and the dust properties can affect, directly or indirectly, the cooling because they affect the $\mathrm{CO}$ excitation. Some general characteristics of the $\mathrm{CO}$ radiative cooling apply for a range of parameters and can be summarized as follows. First, very high excitation lines ( $J$ around 25$)$ need to be considered in the thermal balance because they are relevant in the inner region of the envelope; even if their level populations are low, the cooling contribution of these transitions is much higher than that of lower excitation transitions. Second, the submillimeter lines, such as $J=6 \rightarrow 5$, are very important for constraining the temperature at a radius around $10^{16} \mathrm{~cm}$, because they dominate the cooling in this region. In contrast, low-excitation lines such as $J=2 \rightarrow 1$ and $J=1 \rightarrow 0$ do not provide much information about this region because they contribute little to the cooling. These lines, however, dominate in the outer part of the envelope, at $r \simeq 10^{17} \mathrm{~cm}$, so that their radial distribution can help us to determine the temperature around this radius. This pattern in the radiative cooling can be seen in Figure 1, where the contribution of each transition to the cooling is plotted as a function of distance from the star. For each line, the flat part of the curve corresponds to the optically thick regime, in which the cooling tends to be the same as a blackbody of temperature equal to the excitation temperature of the transition (Goldsmith \& Langer 1978). The curves start to drop at a different radius for each transition, where they become less optically thick because of the decrease in density with increasing radius. In

TABLE 1

OBSERVATIONS

\begin{tabular}{clcl}
\hline \hline Line & Telescope & $\begin{array}{c}\text { Beam Size } \\
(\operatorname{arcsec})\end{array}$ & \multicolumn{1}{c}{ References } \\
\hline${ }^{12} \mathrm{CO} J=1 \rightarrow 0 \ldots \ldots \ldots$ & IRAM & 21 & Truong-Bach et al. 1991 \\
& NRAO & 50 & Huggins et al. 1988 \\
& BTL & 106 & Kwan \& Linke 1982 \\
${ }^{12} \mathrm{CO} J=2 \rightarrow 1 \ldots \ldots \ldots$ & IRAM & 12 & Kahane et al. 1992; Truong-Bach et al. 1991 \\
& NRAO & 31 & Huggins et al. 1988 \\
${ }^{12} \mathrm{CO} J=3 \rightarrow 2 \ldots \ldots \ldots$ & JCMT & 15 & Williams \& White 1992 \\
& CSO & 20 & Wang et al. 1994 \\
${ }^{12} \mathrm{CO} J=4 \rightarrow 3 \ldots \ldots \ldots$ & JCMT & 11 & Williams \& White 1992 \\
${ }^{12} \mathrm{CO} J=6 \rightarrow 5 \ldots \ldots \ldots$ & JCMT & 7 & This work \\
& CSO & 10 & This work \\
${ }^{12} \mathrm{CO} J=9 \rightarrow 8 \ldots \ldots \ldots$ & KAO & 73 & Skinner et al. 1995 \\
${ }^{12} \mathrm{CO} J=12 \rightarrow 11 \ldots \ldots$. & KAO & 55 & Skinner et al. 1995 \\
${ }^{13} \mathrm{CO} J=1 \rightarrow 0 \ldots \ldots .$. & IRAM & 21 & Mauersberger et al. 1989 \\
${ }^{13} \mathrm{CO} J=3 \rightarrow 2 \ldots \ldots \ldots$ & CSO & 21 & Wang et al. 1994 \\
${ }^{13} \mathrm{CO} J=6 \rightarrow 5 \ldots \ldots \ldots$ & CSO & 10 & This work \\
\hline
\end{tabular}




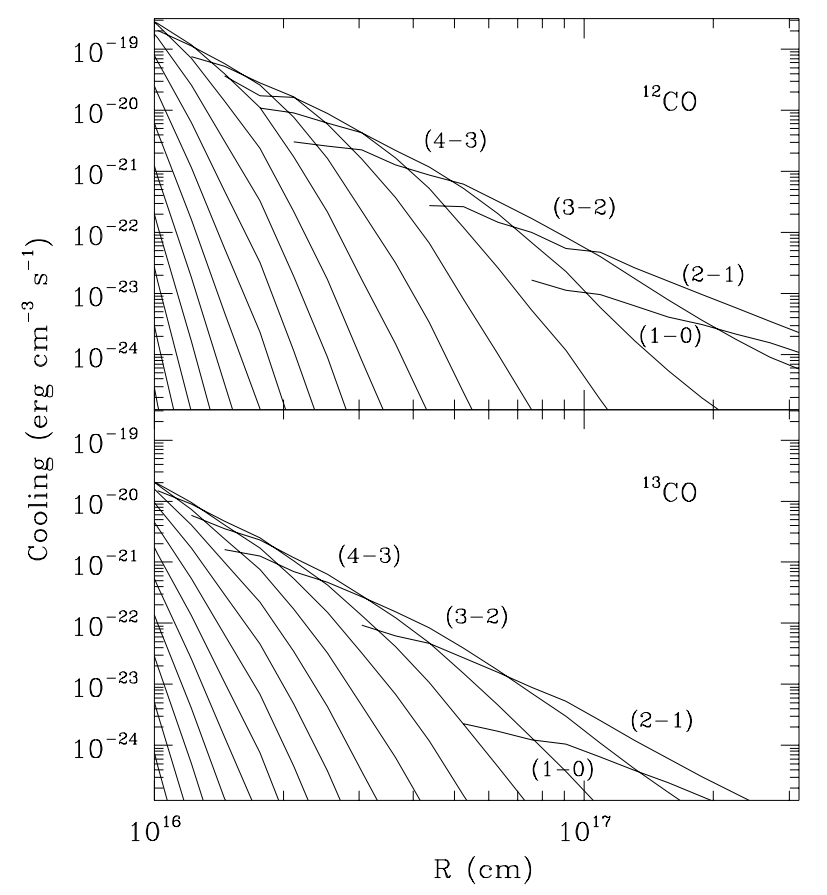

FIG. 1.- Radiative cooling produced by ${ }^{12} \mathrm{CO}$ and ${ }^{13} \mathrm{CO}$ rotational transitions as a function of radial distance from the star.

the hottest region of the envelope, the maximum cooling is produced by transitions with $J$ between 25 and 30. Lines with higher $J$ values become optically thin because of low populations in these rotational levels, and their contribution to the cooling becomes rapidly negligible as $J$ increases.

Although ${ }^{12} \mathrm{CO}$ is the dominant coolant throughout the envelope, ${ }^{13} \mathrm{CO}$ contributes significantly in some regions. In the innermost part, around $r \sim 10^{15} \mathrm{~cm}$, the ${ }^{12} \mathrm{CO}$ cooling is about 50 times larger than the ${ }^{13} \mathrm{CO}$ cooling (it is equal to the $\left[{ }^{12} \mathrm{CO} /{ }^{13} \mathrm{CO}\right]$ abundance ratio; see next section), because the lines dominating the cooling are optically thin. At $r \sim 5 \times 10^{15} \mathrm{~cm}$, the $J=12 \rightarrow 11$ line dominates the cooling. This line is slightly optically thick, so the ratio between the ${ }^{12} \mathrm{CO}$ and the ${ }^{13} \mathrm{CO}$ cooling rates is lower than the abundance ratio, $\sim 20$. At $r \sim 10^{16} \mathrm{~cm}$, the $J=6 \rightarrow 5$ line dominates the cooling, and since this line is more optically thick than the previous ones, the ratio between the ${ }^{12} \mathrm{CO}$ and the ${ }^{13} \mathrm{CO}$ cooling rates is even lower, about 16 . This ratio stays the same up to $r \sim 5 \times 10^{16} \mathrm{~cm}$, where the $J=3 \rightarrow 2$ line dominates the cooling. Then the ratio between cooling rates increases again to reach a value of 50 , as the lines that dominate the cooling $(J=3 \rightarrow 2$ and $J=2 \rightarrow 1$ ) become more optically thin in the outer part of the envelope.

\subsection{Model Parameter Results}

Our model calculations yield CO line intensities and profiles for various impact parameters. These model spectra are then convolved with a Gaussian beam profile (two Gaussians for the ${ }^{12} \mathrm{CO} J=6 \rightarrow 5$ line taken with the JCMT) to allow direct comparison with the main-beam brightness temperature, $T_{\mathrm{MB}}$, detected by a (sub)millimeter telescope (see, e.g., Wang et al. 1994 for a discussion of submillimeterwavelength calibration issues). Good agreement between the model results and the observations is obtained using the following set of parameters: a mass-loss rate, $\dot{M}$, of
$3.25 \times 10^{-5} M_{\odot} \mathrm{yr}^{-1},\left[\mathrm{CO} / \mathrm{H}_{2}\right]=6 \times 10^{-4}$, a dust-gas momentum transfer efficiency, $Q$, of $2.5 \times 10^{-2}$ for the inner region $\left(r \leq 10^{16}\right)$ and $1.8 \times 10^{-2}$ for the outer region $\left(r>10^{16}\right)$, and a distance, $d$, of $150 \mathrm{pc}$.

In Figure 2, observed and predicted emission line profiles are shown for the ${ }^{12} \mathrm{CO} J=1 \rightarrow 0, J=2 \rightarrow 1, J=3 \rightarrow 2$, $J=4 \rightarrow 3$, and $J=6 \rightarrow 5$ transitions. Our model reproduces very well the shape of the observed lines, and the calculated intensities are also in good agreement with the observed values, if we take into account that the absolute

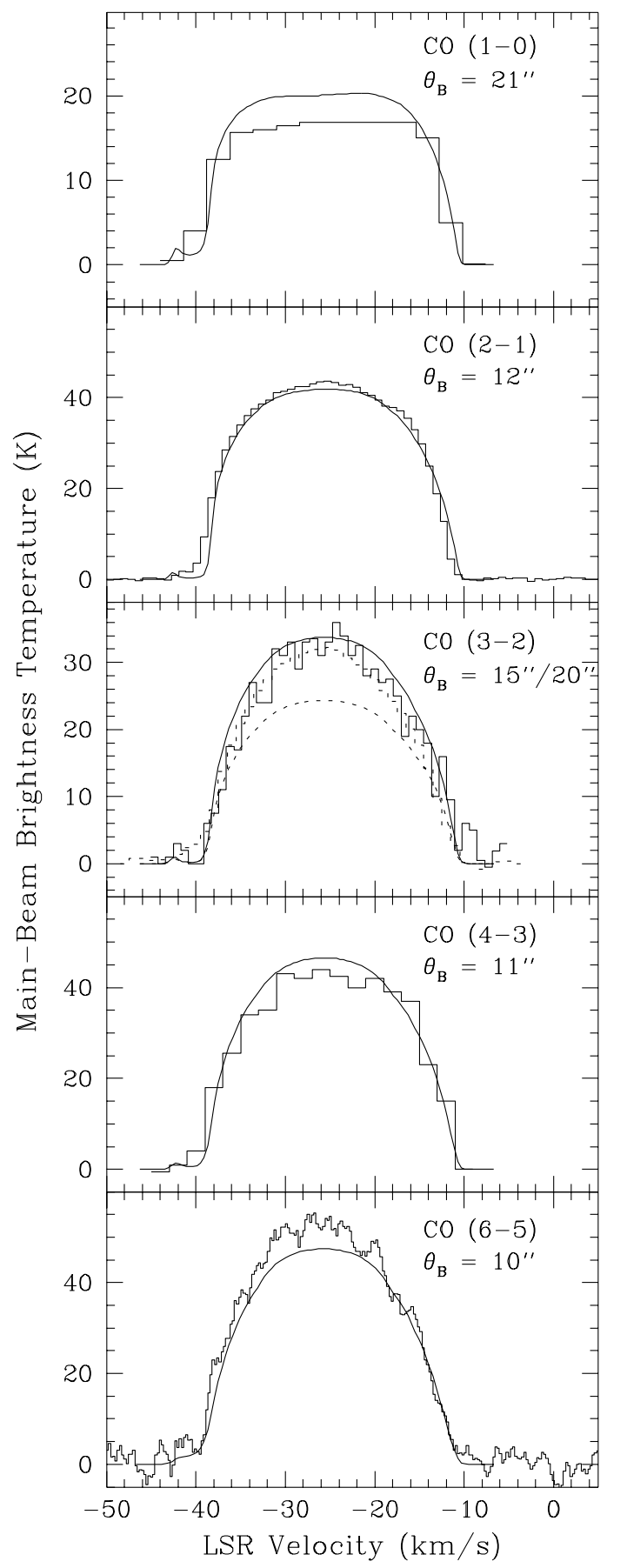

FIG. 2.-Observed line profiles on-source for CO $J=1 \rightarrow 0, J=2 \rightarrow 1$, $J=3 \rightarrow 2, J=4 \rightarrow 3$, and $J=6 \rightarrow 5$ (histograms) compared with model results (solid lines) using the parameters given in text. The beam sizes are indicated in the figure. For $\mathrm{CO} J=3 \rightarrow 2$, we show the observed and theoretical line profiles for two beam sizes: a $15^{\prime \prime}$ beam (solid line) and a $20^{\prime \prime}$ beam (dotted line). 


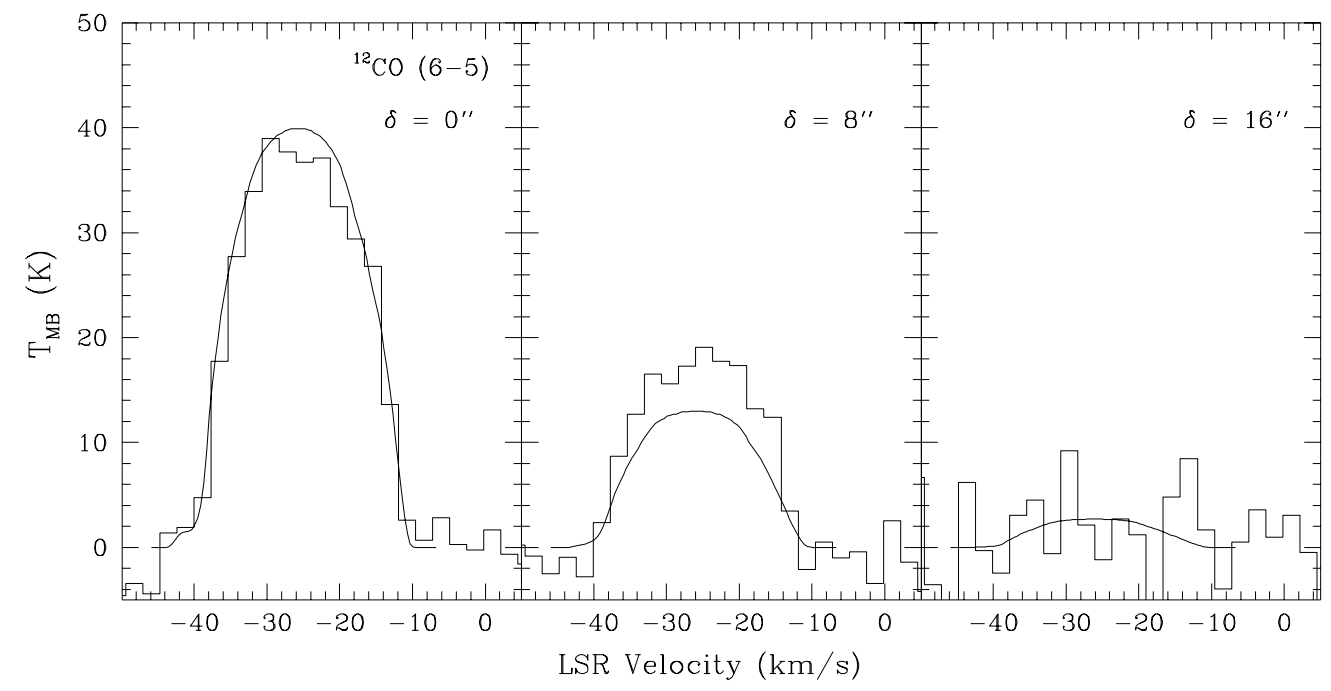

FIG. 3.-Observed (histograms) and theoretical (solid lines) line profiles for ${ }^{12} \mathrm{CO} J=6 \rightarrow 5$ at $0^{\prime \prime}, 8^{\prime \prime}$, and $16^{\prime \prime}$ from the central star, measured with the JCMT.

calibration at (sub)millimeter wavelengths usually has errors of order $10 \%-20 \%$. For the CO $J=3 \rightarrow 2$ transition observed with a $20^{\prime \prime}$ beam, corresponding to the CSO value, the model predicts a peak $T_{\mathrm{MB}}$ of $\approx 26 \mathrm{~K}$. This is within the uncertainties that are consistent with the main-beam brightness temperatures measured for this line with the CSO by Wang et al. (1994) and Young (1995), who report values of 32 and $29 \mathrm{~K}$, respectively, both of which are significantly smaller than the $\approx 44 \mathrm{~K}$ reported by Groesbeck et al. (1994). We prefer the former, lower values, since calibration issues were a central concern of the Wang et al. paper. For the ${ }^{12} \mathrm{CO} J=6 \rightarrow 5$ spectrum obtained with the CSO, the line intensity is very sensitive to the beam size because the $10^{\prime \prime}$ CSO beam matches the size of the region from which the bulk of the $J=6 \rightarrow 5$ emission arises. For example, convolving with a $11^{\prime \prime}$ rather than a $10^{\prime \prime}$ FWHM beam would decrease the main-beam brightness temperature at line center by $12 \%$. For the same reason, the line intensity is sensitive to pointing errors also; an offset of about $2^{\prime \prime}$ produces a decrease of $6 \%$. In Figure 3, we show the JCMT ${ }^{12} \mathrm{CO} J=6 \rightarrow 5$ spectra for the $0^{\prime \prime}, 8^{\prime \prime}$, and $16^{\prime \prime}$ offset positions. Again, the line shapes are well reproduced for this case. The model intensity of the center position, $8^{\prime \prime}$, is slightly lower than the observed intensity. Figure 4 shows the comparison between the observed and the predicted radial distributions for ${ }^{12} \mathrm{CO} \quad J=1 \rightarrow 0, \quad J=2 \rightarrow 1, \quad$ and $J=3 \rightarrow 2$. The predicted main-beam brightness temperature for ${ }^{12} \mathrm{CO} J=1 \rightarrow 0$ is slightly overestimated in the central region, but the disagreement is less than $20 \%$, which is within the calibration uncertainties. The agreement improves at outer radii (or with larger beam telescopes). The maps for $\mathrm{CO} J=2 \rightarrow 1$ and $\mathrm{CO} J=3 \rightarrow 2$ show good agreement between model and observations.

The ${ }^{13} \mathrm{CO} J=1 \rightarrow 0$ line is optically thin at offsets of $0^{\prime \prime}$, $10^{\prime \prime}$, and $20^{\prime \prime}$ from the central source. Figure 5 shows that our model can accurately reproduce the double-peaked profile and intensity of this line. The isotopic abundance is taken to be 1/50 times the $\mathrm{CO}$ abundance, a value consistent with previous studies (Kahane et al. 1992). Finally, Figure 6 shows the $\mathrm{CSO}{ }^{13} \mathrm{CO} J=3 \rightarrow 2$ and $J=6 \rightarrow 5$ transitions. Using ${ }^{12} \mathrm{CO} /{ }^{13} \mathrm{CO}=50$, the model intensity is somewhat lower than that observed for ${ }^{13} \mathrm{CO} J=6 \rightarrow 5$.
The agreement improves by using ${ }^{12} \mathrm{CO} /{ }^{13} \mathrm{CO}=45$, which increases slightly the ${ }^{13} \mathrm{CO} J=1 \rightarrow 0$ model line intensity. However, the poor fit of the ${ }^{13} \mathrm{CO} J=6 \rightarrow 5$ model profile to the data may be the result of a blend of an unidentified line with the ${ }^{13} \mathrm{CO}$ line.

We have also compared our model results with recent observations of ${ }^{12} \mathrm{CO} J=9 \rightarrow 8$ and ${ }^{12} \mathrm{CO} J=12 \rightarrow 11$ made with the Kuiper Airborne Observatory (KAO) (Skinner et al. 1995). These are the highest transitions observed toward AGB stars, and they are of interest for the study of the inner region, $r \sim 10^{15} \mathrm{~cm}$, of the envelope. Their observed main-beam brightness temperature is $1.0 \mathrm{~K}$ with a beam of size $73^{\prime \prime}$ for ${ }^{12} \mathrm{CO} J=9 \rightarrow 8$, and $1.1 \mathrm{~K}$ with a beam of size $55^{\prime \prime}$ for ${ }^{12} \mathrm{CO} J=12 \rightarrow 11$. The poor signalto-noise ratio and the large beam sizes of these observations do not allow a detailed modeling of the line profiles. However, we can compare the observed and the model brightness temperature at the line center. Our model gives a main-beam brightness temperature of $0.8 \mathrm{~K}$ for ${ }^{12} \mathrm{CO}$ $J=9 \rightarrow 8$, and of 0.9 for ${ }^{12} \mathrm{CO} J=9 \rightarrow 8$, which is in good agreement with the observations.

CO lines from levels with $J \geq 14$ have been observed recently with the ISO (Cernicharo et al. 1996). The fluxes of these lines depend strongly on the kinetic temperature in the innermost part of the envelope, $r \leq 10^{15} \mathrm{~cm}$. Our model does not take into account all the physical processes that can be important in this inner region, such as $\mathrm{HCN}$ cooling, grain formation, and shocks. Therefore, its application to fit the observed line profiles of these very high excitation transitions is not appropriate. However, we note that our temperature near $r_{\min }=5 \times 10^{14} \mathrm{~cm}$ of around $1200 \mathrm{~K}$ is consistent with the temperature needed to reproduce, within a factor of 2 , the measured intensities of the transitions involving levels with $J \geq 14$. Since these lines are very optically thick, their intensities do not depend significantly on the amount of material inside our minimum radius.

\section{DISCUSSION}

There is small flexibility for the model parameter values once the distance is fixed, although it is difficult to prove the uniqueness of a given set of best-fit parameters. We discuss 


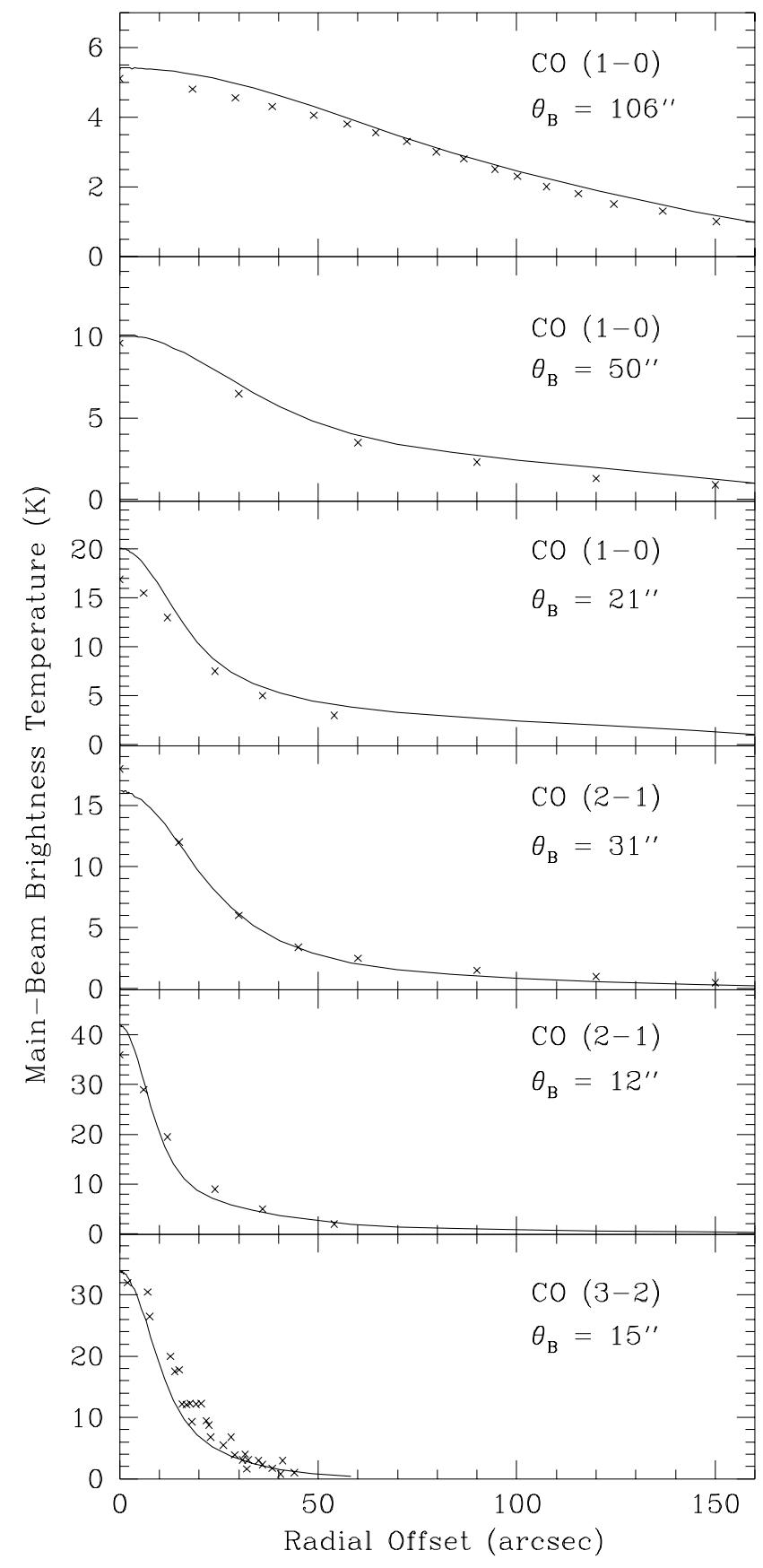

FIG. 4.-Radial distribution of $\mathrm{CO} \quad J=1 \rightarrow 0, \quad J=2 \rightarrow 1$, and $J=3 \rightarrow 2$ at line center, observed with various telescopes (beam sizes are indicated for each case). The data values are shown as crosses, and the solid lines correspond to our model results.

the effects caused by variations of the input model parameters and justify our choices.

\subsection{Distance to the Source}

In particular, our best estimate for the distance to IRC $+10216,150 \mathrm{pc}$, is constrained by the CO $J=1 \rightarrow 0$ and CO $J=2 \rightarrow 1$ maps. This distance has been suggested by other recent studies also (Zuckerman, Dyck, \& Claussen 1986; Kastner 1992). A larger distance would require a higher infrared luminosity and a higher mass-loss rate. Thus, we cannot reproduce exactly the same line profiles with different distances, because the line intensities are affected by changes not only in the radiative transfer but also in the thermal balance. However, models with different distances can give similar results that still might be acceptable within the uncertainties of the observations, if the appropriate scaling in mass-loss rate and luminosity is applied. The luminosity varies as $d^{2}$. If the mass-loss rate is varied as $d$, our model results for $d \leq 200 \mathrm{pc}$ are still acceptable. At larger distances, the disagreement between model results and observations increases. The resulting kinetic temperature increases more in the outer region than in the inner region of the envelope. Thus, the low-excitation $\mathrm{CO}$ $J=1 \rightarrow 0$ and $J=2 \rightarrow 1$ lines, for which the bulk of the emission comes from between radii of $5 \times 10^{16}$ and $3 \times 10^{17} \mathrm{~cm}$, become too bright compared with the observations, with brightness temperatures more than $20 \%$ higher than the observed values, because of the high temperature in this region. Higher excitation lines, which originate in a smaller region $\left(r \leq 5 \times 10^{16} \mathrm{~cm}\right)$, are less affected by such a temperature increase. As a solution to this disagreement, we find that the line intensities can be kept almost constant when we increase the distance if we also vary $Q$ as $d^{-1 / 2}$. This is in agreement with a more general study done by Kwan \& Webster (1993). They define a parameter $q$ that depends on $L^{3 / 2}$ and $Q^{3 / 2}$. They suggest that this parameter should be $q \approx 10_{-5}^{+10}(d / 200 \mathrm{pc})^{2.3}$ for IRC +10216 . This corresponds to a dependence of $Q$ on the distance of $d^{-1 / 2}$. Our value for $q$ is about 10 for $150 \mathrm{pc}$, which is consistent with their value within the uncertainties. Their study also suggests a mass-loss rate varying as $d$. However, even though their scaling works well for line profiles and intensities on-source, we find that it does not work well for the radial distributions of the $\mathrm{CO} J=1 \rightarrow 0$ and CO $J=2 \rightarrow 1$ intensities. For that reason, we favor a low distance, $d \sim 150 \mathrm{pc}$, because it produces the best fit to the observed line profiles and the radial distributions at the same time.

\subsection{Dust-to-Gas Momentum Transfer Efficiency Factor}

Changes in the $Q$-parameter affect the heating curve directly. The heating is proportional to $Q^{2 / 3}$ in most of the envelope, except in the innermost, acceleration region, where the thermal velocity is comparable to the velocity resulting from acceleration by radiation pressure (see eqs. [6], [7], and [8]).

We find that the high-excitation emission originating in the inner region is better reproduced with a higher value of $Q, 2.5 \times 10^{-2}$, than that for the more extended and lower excitation emission, where $Q=1.8 \times 10^{-2}$. This behavior of $Q$ agrees with recent radiative transfer studies such as that of Habing et al. (1994), which suggest that the value of $Q$ decreases with distance from the star for carbon-rich circumstellar envelope. This drop in $Q$ occurs mainly because the absorption efficiency of carbonaceous dust grains decreases as the effective wavelength of the stellar radiation increases with radial distance. In their study, $Q$ has high values, $(2-6) \times 10^{-2}$, in a very small inner region, $r \leq 10^{15}$ $\mathrm{cm}$, and then it drops very rapidly with radial distance from the star and reaches a practically constant value at the outer region. Our "inner" region is somewhat larger than theirs, but the overall characteristics are the same. Introducing a continuous variation of $Q$ instead of our discontinuity at $10^{16} \mathrm{~cm}$ does not modify the results significantly. Our low $Q$ value, $1.8 \times 10^{-2}$, in the outer region is similar to their mean value throughout this same region.

The dust-to-gas mass ratio also affects the heating directly. In our model, its value is fixed at $10^{-2}$, the value 


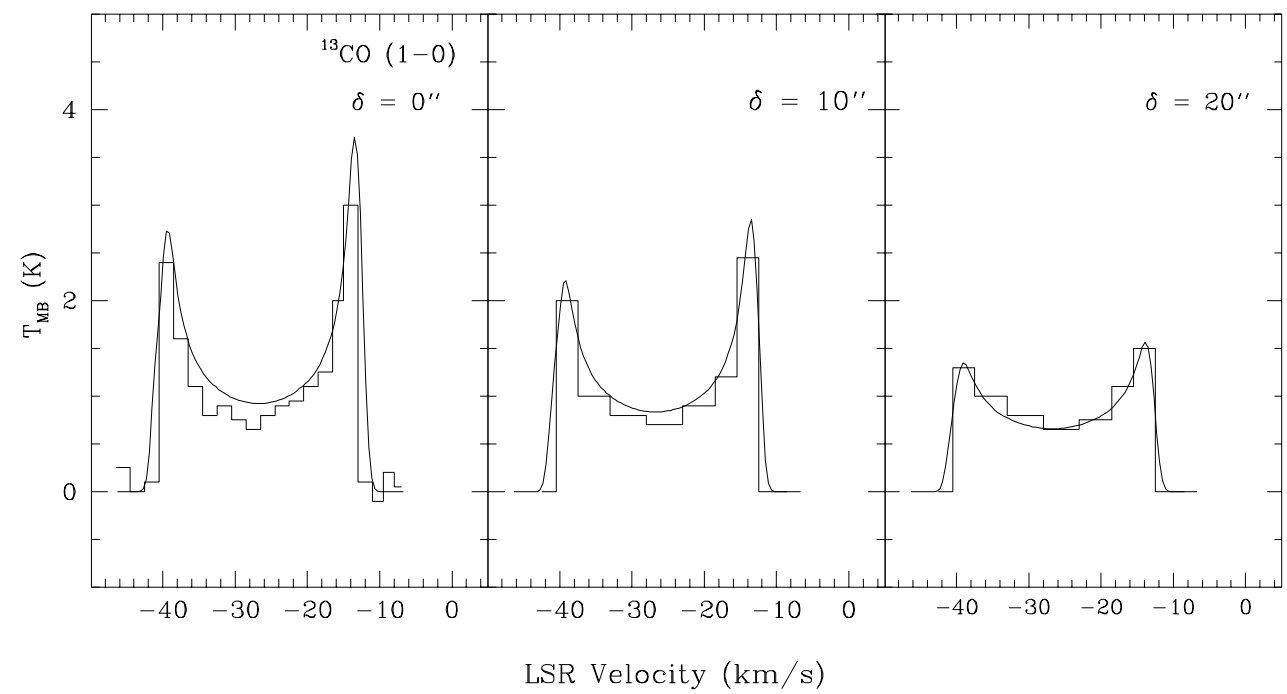

FIG. 5.-Observed (histograms) and theoretical (solid lines) line profiles for ${ }^{13} \mathrm{CO} J=1 \rightarrow 0$ at $0^{\prime \prime}$, $10^{\prime \prime}$, and $20^{\prime \prime}$ from the central star, obtained with the IRAM $30 \mathrm{~m}$ telescope.

typical for the ISM. It has been suggested by recent studies of the dust mass-loss rate in IRC +10216 that this ratio could be as low as $1.5 \times 10^{-3}$ (Ivezić \& Elitzur 1996). However, using this low value would result in an extreme reduction of the heating, yielding line intensities that are consistently too low. A better match to the observations could be achieved by increasing $Q$ by a factor of 3, resulting in a value that is not consistent with studies of dust properties (e.g., Habing et al. 1994). For example, by increasing the gas mass-loss rate, we would not achieve good agreement between model and observed line profiles, because the lines would become too optically thick.

\subsection{Mass-Loss Rate and $\mathrm{CO}$ Abundance}

The mass-loss rate and the $\mathrm{CO}$ abundance affect the radiative transfer in a similar way, because the number of $\mathrm{CO}$ molecules depends on the total density, which is pro-

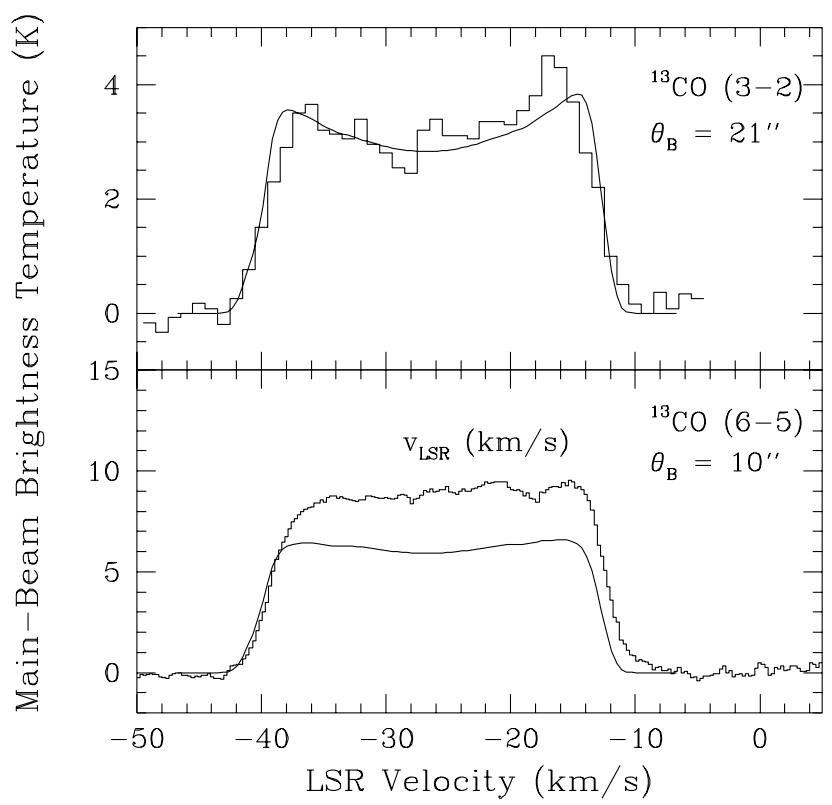

Fig. 6.-Observed (histograms) and theoretical (solid lines) line profiles on-source for ${ }^{13} \mathrm{CO} J=3 \rightarrow 2$ and $J=6 \rightarrow 5$, measured with the CSO. portional to the mass-loss rate. If the lines are optically thin, the intensity scales with the CO column density. Even if the observed profiles of a given $\mathrm{CO}$ transition indicate optically thick emission, the line might be optically thin in significant portions of the envelope, and thus dependent on $\mathrm{CO}$ density. However, if increasing the density causes lines to become optically thick throughout the envelope, the resulting effect on the radiative transfer is almost negligible. $\mathrm{CO} J=1 \rightarrow 0$ is affected particularly by changes in the $\mathrm{CO}$ abundance and density because it is less optically thick than the other observed CO lines, which are practically unaffected.

It is important to note that varying $\dot{M}$ and $\left[\mathrm{CO} / \mathrm{H}_{2}\right]$ affects the thermal balance in different ways. Variations of the CO-to- $\mathrm{H}_{2}$ abundance ratio influence the radiative cooling only. Increasing the mass-loss rate increases the heating in the whole envelope, while the cooling in the inner envelope remains unchanged since the density in this region is high and the CO lines are thermalized. With increasing distance from the star, the cooling may increase (more than the heating) because the $\mathrm{CO}$ lines are no longer thermalized and optically thick, and there are more molecules to produce cooling. Thus, the net effect of an increase in the mass-loss rate is a temperature increase in the inner region and a small decrease in the outer region. In contrast, an increase in $\mathrm{CO}$ abundance does not produce additional heating in the inner region. It only causes a temperature decrease in the outer region. This means that we cannot reproduce exactly the results in the $\mathrm{CO}$ emission by varying the $\mathrm{CO}$ abundance proportionally to the mass-loss rate.

Choosing a value for $Q$ that is lower than our best value requires a higher mass-loss rate to reproduce the observed line intensities, which worsens the agreement between the model and the observed radial distributions for $\mathrm{CO}$ $J=1 \rightarrow 0, \mathrm{CO} J=2 \rightarrow 1$, and $\mathrm{CO} J=3 \rightarrow 2$. As described above, an increase in the gas density can increase the temperature in the inner region. Then, although the intensity and on-source line profiles might be well reproduced for most lines, the line emission drops too rapidly with radius because of the sudden drop in temperature. This behavior in the temperature affects mostly the $\mathrm{CO} J=3 \rightarrow 2$ line because the temperature gradient is steeper in the regions 
from which the bulk of the emission in this line arises, within $r \sim 5 \times 10^{16} \mathrm{~cm}$ from the center.

\subsection{Excitation by the Infrared Field}

We explore the effect of extended infrared emission at $r=1^{\prime \prime}$, in addition to the blackbody radiation field from the inner $r \approx 0$ ".2 dust shell (see $\S 2.2$ ). We use a blackbody radiation field with $T=375 \mathrm{~K}$. The total flux at $4.6 \mu \mathrm{m}$ obtained from the two blackbodies is an upper limit to the flux at a radial distance from the star of about $1^{\prime \prime}$, so we use it to prove that the infrared emission originating in this inner region of the envelope does not effect the $\mathrm{CO}$ emission, regardless of the details of the distribution of such emission. In fact, our calculations show that the $\mathrm{CO}$ and ${ }^{13} \mathrm{CO}$ line profiles remain exactly the same after adding this second component. This is because the emitting region is too small compared with the beam size of the telescopes, and in addition the flux at $4.6 \mu \mathrm{m}$ from this component is not large enough to produce significant changes in the excitation temperature of the observed $\mathrm{CO}$ lines.

We now consider whether other effects of infrared pumping could be important in our model calculations. We first consider excitation to the $v=2$ state. We examine the effect of the $2.2 \mu \mathrm{m}$ continuum radiation that is capable of exciting $\mathrm{CO}$ molecules from the ground state directly to the $v=2$ state. We find that the relatively low value of the relevant Einstein $A$-coefficients $\left(A \approx 0.5 \mathrm{~s}^{-1}\right.$ compared with $20-30 \mathrm{~s}^{-1}$ for the $A$-values of $v=1 \rightarrow 0$ transitions) and the lower infrared flux at this wavelength (about 10 times less than at $4.6 \mu \mathrm{m}$ ) make direct $v=0 \rightarrow 2$ excitation negligible. Second, we consider excitation to the $v=2$ state through the $v=1$ state. In this case, the $A$-values for $v=2 \rightarrow 1$ are comparable to the $v=1 \rightarrow 0$ values, and both transitions can be excited by $4.6 \mu \mathrm{m}$ radiation. We find that the process of pumping the molecules from $v=1$ to $v=2$ shifts the rotational population to higher $J$ levels. The term $R_{J, J+2}$ defined in $\S 2.2$ can be increased by a factor of 2 by this process. In addition, there is another term, $R_{J, J+4}$, that can increase the excitation to the higher $J$ level by a factor of 4 or more. (The inverse terms, which produce de-excitations, i.e., from $J$ to $J-4$, are always lower because of the values of the $A$-coefficients and statistical weights.) But again, this process, although it can be important in some cases, is not significant in our case because $\mathrm{CO}$ and ${ }^{13} \mathrm{CO}$ are too optically thick to the $4.6 \mu \mathrm{m}$ continuum. That is, it is only important in a very small inner region where the infrared flux is not yet absorbed, so it does not modify our results. We also examine the effect of the acceleration region on the infrared pumping. In this region, there is no attenuation in radial direction; therefore, the total infrared absorption by $\mathrm{CO}$ is reduced. For an acceleration region of size $r \sim 3 \times 10^{14} \mathrm{~cm}$ (as we assumed in our model), there is no change in the results. The effect starts to be noticeable if the acceleration region has a size of about $5 \times 10^{15} \mathrm{~cm}$. Then the ${ }^{12} \mathrm{CO} J=6 \rightarrow 5$ and $J=4 \rightarrow 3$ line intensities increase by $10 \%$, but the lower excitation lines and ${ }^{13} \mathrm{CO}$ lines are affected minimally. This is due to the fact that the intensities of the high-excitation ${ }^{12} \mathrm{CO}$ lines increase proportionally to the excitation temperature $T_{\mathrm{ex}}$ since they are optically thick. However, the ${ }^{13} \mathrm{CO} J=6 \rightarrow 5$ line intensity does not increase because it is optically thin, so that $\tau \propto 1 / T_{\text {ex }}$, which cancels the $T_{\mathrm{ex}}$ dependence. Since the acceleration region is unlikely to be larger than $5 \times 10^{15} \mathrm{~cm}$, our results are not affected significantly.

\subsection{Photodissociation}

We assumed that photodissociation starts destroying both ${ }^{12} \mathrm{CO}$ and ${ }^{13} \mathrm{CO}$ at the same radius $\left(3.75 \times 10^{17} \mathrm{~cm}\right.$, for $d=150 \mathrm{pc}$ ), despite the fact that ${ }^{13} \mathrm{CO}$ has a larger photodissociation rate because it is not protected by selfshielding. Mamon et al. (1988) claim that ${ }^{13} \mathrm{CO}$ is replenished by the fractionation reaction

$$
{ }^{12} \mathrm{CO}+{ }^{13} \mathrm{CO}^{+} \rightarrow{ }^{13} \mathrm{CO}+{ }^{12} \mathrm{CO}^{+}
$$

and suggest that for IRC +10216 , the two effects conspire to make the radii at which ${ }^{12} \mathrm{CO}$ and ${ }^{13} \mathrm{CO}$ are photodissociated of similar size. As a check, we have performed calculations with different ${ }^{13} \mathrm{CO}$ radii. We find that, using $r=2 \times 10^{17} \mathrm{~cm}$ for the ${ }^{13} \mathrm{CO}$ photodissociation radius, the observed ${ }^{13} \mathrm{CO} J=1 \rightarrow 0$ line profiles are better reproduced than with $r=3.75 \times 10^{17} \mathrm{~cm}$. In particular, the peak-to-line center contrast is smaller, in agreement with observations. Choosing an even smaller ${ }^{13} \mathrm{CO}$ photodissociation radius results in line profiles that are too flat and disagree substantially with the observations.

\subsection{Kinetic Temperature: Comparison with Previous Works}

Depending on their excitation requirements, various $\mathrm{CO}$ lines have the bulk of their observed emission arising from distinct parts of the envelope. In particular, the high spatial resolution observation of the $\mathrm{CO} J=6 \rightarrow 5$ transition helps to constrain the temperature in the inner region $\left(r \approx 5 \times 10^{15} \mathrm{~cm}\right)$, which has not been well probed by previous observations.

The kinetic temperature as a function of radius obtained by our model is shown in Figure 7, in which we compare our result with the temperature profiles obtained by Kwan \& Linke (1982) and by Truong-Bach et al. (1991), who also performed a self-consistent calculation of the thermal balance. Our curve is very different from that of TruongBach et al. (1991) because they use a two-shell model with different mass-loss rates, which causes an abrupt temperature discontinuity at the boundary between the shells. In particular, these authors suggest the existence of a very hot inner region of temperature greater than $500 \mathrm{~K}$ extending out to a radius of $10^{16} \mathrm{~cm}$. Their model was motivated

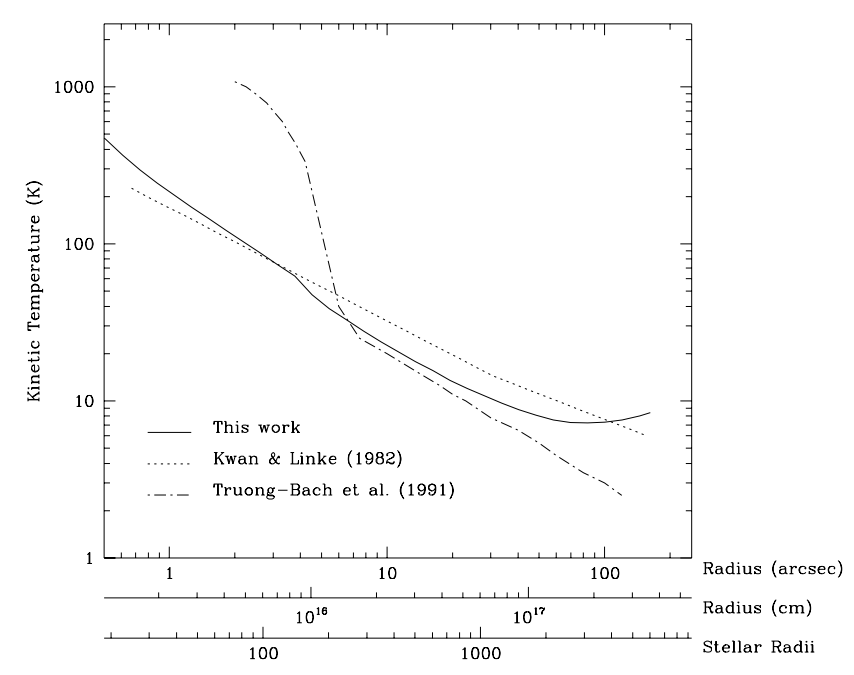

FIG. 7.-Kinetic temperature as a function of radial distance from the star for our model, compared with Kwan \& Linke (1982) and Truong-Bach et al. (1991) models. 
primarily by a low-resolution ( $\left(35^{\prime \prime}\right) \mathrm{CO} J=6 \rightarrow 5$ spectrum, whose intensity may have been overestimated by a factor of $\approx 2$ because of calibration uncertainties. Using their temperature profile, we have performed statistical equilibrium calculations that result in CO $J=6 \rightarrow 5$ intensities overestimating the values measured with CSO and JCMT by factors of $\approx 3$. Other intermediate-excitation lines (CO $J=4 \rightarrow 3$ and $\mathrm{CO} J=3 \rightarrow 2$ ) are overestimated as well. One of the major reasons for the very different physical parameters obtained by Truong-Bach et al. as compared with our results is the fact that their thermal balance calculations consider only 12 rotational levels for $\mathrm{CO}$ and thus underestimate significantly the radiative cooling in the inner envelope, resulting in a much higher temperature in this region. As mentioned above, a consideration of approximately 30 levels is necessary for a proper treatment of the cooling in the region in question.

Our temperature profile for the inner region is similar to that of Kwan \& Linke (1982). However, in order to reproduce the flat line profile of CO $J=1 \rightarrow 0$, we need lower temperatures in the intermediate part of the envelope (between $r \approx 10^{16}$ and $r \approx 10^{17} \mathrm{~cm}$ ) than those obtained from the Kwan \& Linke temperature profile. In their model, the $J=1 \rightarrow 0$ transition is optically thin, and the corresponding line profile double-peaked, contrary to the observations. We obtain slightly higher temperatures in the outer part of the envelope relative to the Kwan \& Linke values by including dust grain photoelectric heating. This results in an improved fit to the radial distributions of the $\operatorname{CO} J=1 \rightarrow 0, J=2 \rightarrow 1$, and $J=3 \rightarrow 2$ transitions.

\section{SUMMARY}

We have calculated the kinetic temperature profile of the IRC +10216 circumstellar envelope by self-consistently solving the thermal balance problem with the radiative transfer, which is treated with a Monte Carlo method. To correctly calculate the radiative cooling in the inner part of the envelope $\left(r<10^{16} \mathrm{~cm}\right)$, we find that it is necessary to include more than 25 rotational levels for $\mathrm{CO}$. Of the existing observational data, recent high spatial resolution $\mathrm{CO}$ $J=6 \rightarrow 5$ data are the most relevant ones to constrain the temperature in this inner region. By comparing the high spatial resolution CO $J=6 \rightarrow 5$ data with our model results, we conclude that there is no need to invoke an extended hot core in the inner envelope as suggested by a previous study. We find that temperatures between 100 and $300 \mathrm{~K}$ in the region between 2 and $8 \times 10^{15} \mathrm{~cm}$ are consistent with the recent submillimeter data. These temperatures are significantly smaller than the values around $1000 \mathrm{~K}$ proposed for this region by a previous study. The temperature in the intermediate and outer part of the envelope is constrained by $\mathrm{CO} J=4 \rightarrow 3, J=3 \rightarrow 2, J=2 \rightarrow 1$, and $J=1 \rightarrow 0$ data.

Our thermal balance/radiative transfer model yields the following set of values for the physical parameters of IRC + 10216: $\dot{M}=3.25 \times 10^{-5} M_{\odot} \mathrm{yr}^{-1},\left[\mathrm{CO} / \mathrm{H}_{2}\right]=$ $6 \times 10^{-4}, \quad Q=2.5 \times 10^{-2}$ for $r \leq 10^{16} \mathrm{~cm}$ and $Q=1.8 \times 10^{-2}$ for $r>10^{16} \mathrm{~cm}$, and $d=150 \mathrm{pc}$. Our massloss rate and $\mathrm{CO}$ abundance values are within the range of typical values from previous studies for the IRC +10216 envelope. We find that the observations are reproduced well by a steady state wind; we do not need to invoke a discrete shells model as it has been suggested in previous studies. The decrease of the dust-to-gas momentum transfer efficiency $Q$ as a function of radial distance from the star in the inner part of the envelope is appropriate for carbonaceous dust grains in carbon-rich envelopes. This behavior helps to reproduce the CO $J=6 \rightarrow 5$ and $\mathrm{CO} J=4 \rightarrow 3$ observations simultaneously. The distance to IRC +10216 has been commonly assumed to be larger than $150 \mathrm{pc}$, but we find that this low value reproduces best the observed $\mathrm{CO}$ $J=2 \rightarrow 1$ and $\mathrm{CO} J=1 \rightarrow 0$ maps.

We would like to thank Mario Tafalla for many useful discussions, and we are very grateful to Andy Harris and Ken Young for providing unpublished submillimeter data.

\section{REFERENCES}

Avery, L. W., et al. 1992, ApJS, 83, 363

Becklin, E. E., Frogel, J. A., Hyland, A. R., Kristian, J., \& Neugebauer, G. 1969, ApJ, 158, L33

Bernes, C. 1978, Stockholm Obs. Rep. 15

. 1979, A\&A, 73, 67

Bieging, J. H., \& Tafalla, M. 1993, AJ, 105, 576

Cernicharo, J., et al. 1996, A\&A, in press

Deguchi, S., \& Uyemura, M. 1984, ApJ, 285, 153

de Jong, T., Chu, S.-I., \& Dalgarno, A. 1975, ApJ, 199, 69

Draine, B. T. 1978, ApJS, 36, 595

Dyck, H. M., Benson, J. A., Howell, R. R., Joyce, R. R., \& Leinert, Ch. 1991, $\mathrm{AJ}, 102,200$

Flower, D. R., \& Launay, J. M. 1985, MNRAS, 214, 271

Goldreich, P., \& Scoville, N. 1976, ApJ, 205, 144

Goldsmith, P. F., \& Langer, W. D. 1978, ApJ, 222, 881

Goorvitch, D., \& Chackerian, C., Jr. 1994, ApJS, 91, 483

Green, S., \& Thaddeus, P. 1976, ApJ, 205, 766

Groenewegen, M. A. T. 1994, A\&A, 290, 531

Groesbeck, T. D., Phillips, T. G., \& Blake, G. A. 1994, ApJS, 94, 147

Habing, H. J., Tignon, J., \& Tielens, A. G. G. M. 1994, A\&A, 286, 523

Harris, A. I., Avery, L. W., Schuster, K.-F., Tacconi, L., \& Genzel, R. 1995, ApJ, 446, L85

Harris, A. I., Schuster, K.-F., Genzel, R., Plathner, B., \& Gundlach, K.-H. 1994, Int. J. Infrared Millimeter Waves, 15, 1465

Huggins, P. J., \& Glassgold, A. E. 1982, ApJ, 252, 201

Huggins, P. J., Olofsson, H., \& Johansson, L. E. B. 1988, ApJ, 332, 1009

Ivezić, Ž., \& Elitzur, M. 1996, MNRAS, 279, 1019

Johansson, L. E. B., et al. 1984, A\&A, 130, 227

Jura, M. 1976, ApJ, 204, 12 1994, ApJ, 434, 713

Jura, M., \& Kleinmann, S. G. 1989, ApJ, 341, 359
Kahane, C., Cernicharo, J., Gómez-González, J., \& Guélin, M. 1992, A\&A, 256,235

Kastner, J. H. 1992, ApJ, 401, 337

Kawaguchi, K., Kasai, Y., Ishikawa, S.-I., \& Kaifu, N. 1995, PASJ, 47, 853

Koepf, G. A., et al. 1982, ApJ, 260, 584

Kooi, J. W., Walker, C. K., LeDuc, H. G., Hunter, T. R., Benford, D. J., \& Phillips, T. G. 1994, Int. J. Infrared Millimeter Waves, 15, 477

Kwan, J., \& Hill, F. 1977, ApJ, 215, 781

Kwan, J., \& Linke, R. A. 1982, ApJ, 254, 587

Kwan, J., \& Webster, Z. 1993, ApJ, 419, 674

Kwok, S. 1975, ApJ, 198, 583

Lorenz-Martins, S., \& Lefévre, J. 1993, A\&A, 280, 567

Mamon, G. A., Glassgold, A. E., \& Huggins, P. J. 1988, ApJ, 328, 797

Mauersberger, R., et al. 1989, A\&A, 79, 217

Menten, K. M., \& Young, K. 1995, ApJ, 450, L67

Morris, M. 1980, ApJ, 236, 823

Rouleau, F., \& Martin, P. G. 1991, ApJ, 377, 526

Sahai, R. 1987, ApJ, 318, 809

Skinner, C. J., Justtanont, K., Tielens, A. G. G. M., Betz, A. L., \& Boreiko, R. T. 1995, ASP Conf. Ser. 73, Airborne Astronomy Symp. on the Galactic Ecosystem: From Gas to Stars to Dust, ed. M. R. Haas, J. A. Davidson, \& E. F. Erickson (San Francisco: ASP), 433

Sloan, G. C., \& Egan, M. P. 1995, ApJ, 444, 452

Toombs, R. I., Becklin, E. E., Frogel, J. A., Law, S. K., Porter, F. C., \& Westphal, J. A. 1972, ApJ, 173, L71

Truong-Bach, Morris, D., \& Nguyen-Q-Rieu 1991, A\&A, 249, 435

Wang, Y., Jaffe, D. T., Graf, U. U., \& Evans, N. J., II 1994, ApJS, 95, 503

Williams, P. G., \& White, G. J. 1992, A\&A, 266, 365

Young, K. 1995, ApJ, 445, 872

Zuckerman, B. 1980, ARA\&A, 18, 263

Zuckerman, B., Dyck, H. M., \& Claussen, M. J. 1986, ApJ, 304, 401 